\title{
Nonsurgical Management of Periapical Lesion: A Case Series
}

\author{
Anand V Susila ${ }^{1}$, Almas Janu ${ }^{2}$
}

\begin{abstract}
Aim and objective: The aim of this study is to manage periapical lesions of endodontic origin without invasive surgery. left behind after cleaning and shaping to prevent reinfection. This will ensure lesion reduction or resolution. by Metapex placement in the next visit. After a 3-month interval, when the lesions healed well, obturation was done. to be a more conservative approach.

Keywords: Endodontic management, Intracanal medicament, Lesion management, Periapical lesion.

Journal of Operative Dentistry and Endodontics (2020): 10.5005/jp-journals-10047-0096
\end{abstract}

Background: Though root canal instrumentation along with irrigation greatly reduces the number of bacteria in the infected canals, it is difficult to obtain complete disinfection in all cases. The use of intracanal medicaments helps in reducing or inhibiting the growth of microbes that are

Case descriptions: Four cases have been presented with periapical lesions that were all accessed, cleaned, and shaped in the first visit, followed

Clinical significance: For the treatment of periapical lesions, nonsurgical therapy should be considered as first-line treatment option as it proves

\section{INTRODUCTION}

Microorganisms exert great influence on the development of endodontic lesions, infection-mediated necrosis, and its subsequent spread to the periapical tissue leading to the periapical lesion. ${ }^{1}$ This results due to host defense mechanisms to the microbial attack from the root canal system that involves a dynamic balance between microbial infective factors and host-mediated immune response at the interface of the pulpoperiodontal junction resulting in local inflammation, disorganization of periapical tissue, and resorption of hard tissues. ${ }^{2,3}$ Though the periapical lesion is defensive against microbial attack, they are not self-healing. ${ }^{2}$

The ultimate goal of endodontic therapy is to totally remove the microbes, their byproducts, and pulpal remnants from infected canal space to return it to its normal form and function without surgical interventions. Conservative endodontic treatment procedures without surgery should be the first line of treatment for all inflammatory lesions of endodontic origin. ${ }^{4}$ Studies have reported $94.4 \%$ of total or partial resolution of lesions of endodontic origin with simple nonsurgical treatment under proper infection control. ${ }^{3,5}$ In the event of unsuccessful nonsurgical treatment, surgery is recommended.

\section{BACKGROUND}

Though root canal instrumentation along with irrigation greatly reduces the number of bacteria in the infected canals, it is difficult to obtain complete disinfection in all cases. Microbes left behind after obturation can proliferate into the isthmuses, apical deltas, and dentinal tubules where certain microbes are obstinate to conventional cleaning and shaping and hence cause persistent infections. Hence, intracanal medicaments are placed in canals to control such microbes. ${ }^{6}$ Calcium hydroxide $(\mathrm{CH})$ is the most widely used medication because of its antibacterial activity which can be used in various combinations. Among these combinations, Metapex, which contains $60 \% \mathrm{CH}$ paste and $38 \%$ iodoform in silicone oil, is frequently used. ${ }^{1}$

This article comprises a series of cases reported to the Department of Conservative Dentistry and Endodontics, Madha Dental College

\begin{abstract}
1,2Department of Conservative Dentistry and Endodontics, Madha Dental College and Hospitals, Chennai, Tamil Nadu, India

Corresponding Author: Anand V Susila, Department of Conservative Dentistry and Endodontics, Madha Dental College and Hospital, Chennai,Tamil Nadu, India,Ph:+9175500223977,e-mail:drsusila2000@ gmail.com

How to cite this article: Susila AV, Janu A. Nonsurgical Management of Periapical Lesion: A Case Series. J Oper Dent Endod 2020;5(2): 99-104.

Source of support: Nil

Conflict of interest: None
\end{abstract}

and Hospital, Chennai, with periapical lesions that were nonsurgically managed by placement of Metapex in the root canals.

\section{Case Descriptions}

\section{Case 1}

A 29-year-old male patient reported to the Department of Conservative Dentistry and Endodontics with pain in his lower left posterior teeth region with a history of initiated root canal treatment. Clinical examination showed the presence of a deep carious lesion in 36 (Fig. 1A), and periapical radiograph revealed radiolucency involving the enamel, dentin, and pulp associated with a periapical radiolucency involving both roots of $2 \times 2 \mathrm{~mm}$ in size. Pulp sensibility tests failed to produce any response in the tooth. It was diagnosed as previously initiated root canal treatment with a symptomatic periodontitis in 36 . Nonsurgical endodontic therapy with Metapex was planned.

After access opening under rubber dam isolation, the working length (WL) was determined, and complete biomechanical preparation with rotary ProTaper files was done. All canals were irrigated with $10 \mathrm{~mL}$ each of $1.5 \%$ sodium hypochlorite $(\mathrm{NaOCl})$ followed by saline irrigation, and a final rinse of $17 \%$ ethylenediaminetetraacetic acid (EDTA) was done. Closed dressing was given in the first visit. During the second visit, the patient was asymptomatic. Metapex was placed as an intracanal medicament 

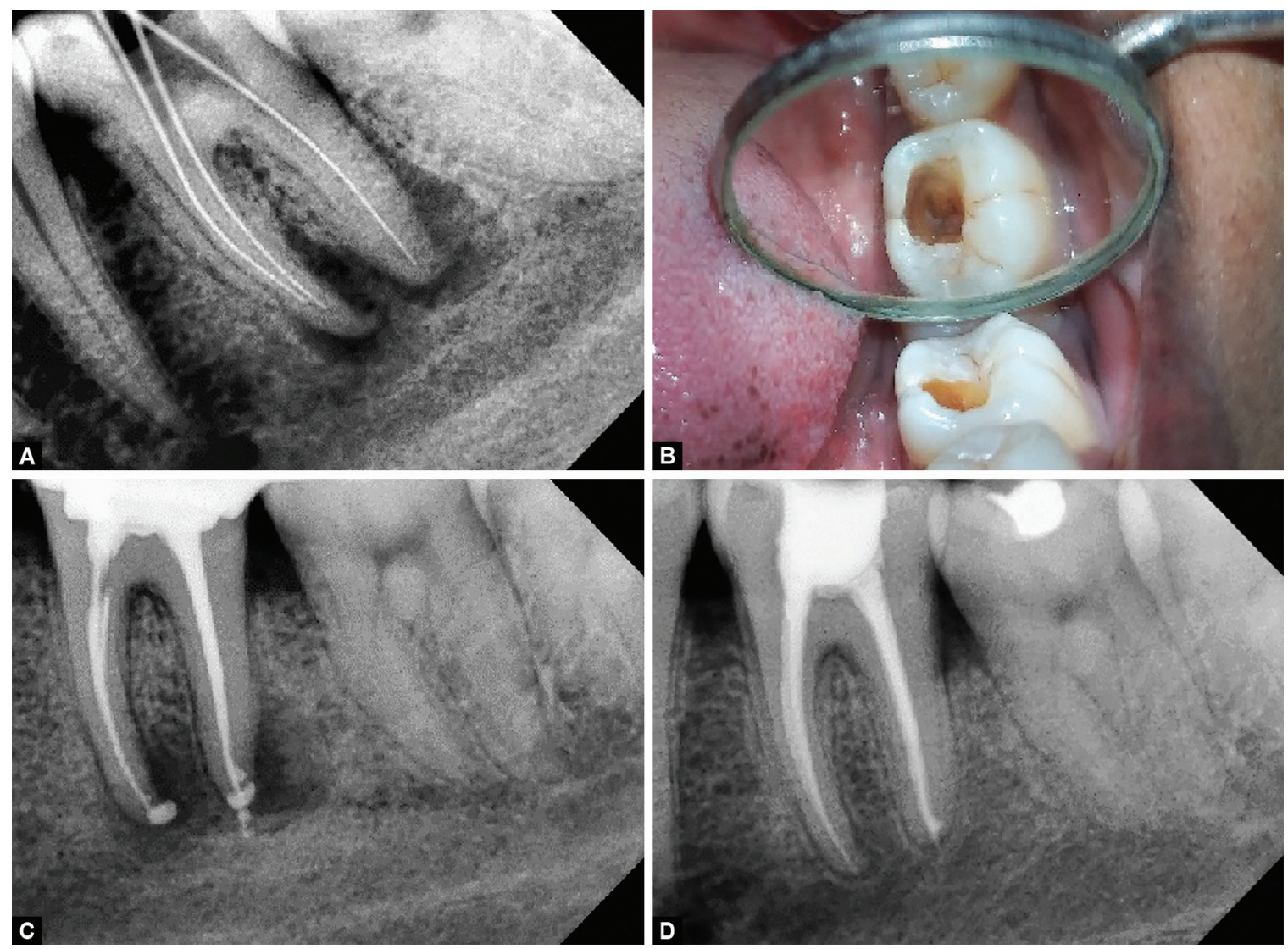

Fig. 1A to D: Case 1: (A) Preoperative clinical photograph; (B) Preoperative intraoral periapical radiograph; (C) Metapex placed, (D) Obturation done

(Fig. 1B). The patient was advised to report after 3 months. After 3 months, resolution of the periapical lesion was evident. So, after Metapex removal from canals, obturation with a single-cone obturation technique was done (Fig. 1D).

\section{Case 2}

A 15-year-old male patient reported to the Department of Conservative Dentistry and Endodontics with pain in his lower left posterior teeth region. The patient had a history of initiated root canal treatment. Clinical examination showed the presence of a broken temporary restoration and gingival swelling in relation to 36 , and periapical radiograph revealed radiolucency involving the enamel, dentin, and pulp associated with a periapical radiolucency involving both roots of $3 \times 3 \mathrm{~mm}$ in size along with furcal involvement (Fig. 2A). Heat and electric pulp tests failed to produce any response in the tooth. It was diagnosed as previously initiated root canal treatment with a periapical abscess in 36 . Incision and drainage of the abscess followed by nonsurgical endodontic therapy with Metapex were planned.

During the first visit, incision of the abscess was done using No 11 BP blade, and the pus was drained after which a rubber drain was placed to aid in complete drainage during the postoperative period. Under rubber dam isolation, access opening followed by complete biomechanical preparation with rotary ProTaper files was done. All canals were irrigated with $10 \mathrm{~mL}$ each of $1.5 \% \mathrm{NaOCl}$ followed by saline irrigation, and an open dressing was given.
During the next visit, complete resolution of the gingival swelling was noticed, and Metapex was placed as an intracanal medicament (Fig. 2B). The patient was advised to report after 3 months. But the patient reported after 5 months during which the healing of the periapical lesion was complete. So, the root canal procedure was completed by obturating the canals with a single-cone obturation technique (Fig. 2D).

\section{Case 3}

A 56-year-old male patient reported to the Department of Conservative Dentistry and Endodontics with pain in his upper left posterior teeth region. Clinical examination showed the intact tooth structures with normal probing depths and healthy gingiva, and IOPA revealed intact enamel and dentin associated with a periapical radiolucency in 24 of $1 \times 2 \mathrm{~mm}$ in size (Fig. 3A). Pulp sensibility tests produced delayed response in the tooth. It was diagnosed as symptomatic irreversible pulpitis with apical periodontitis in 24. Nonsurgical endodontic therapy with Metapex was planned.

After access opening under rubber dam isolation and $\mathrm{WL}$ determination, complete cleaning and shaping of the canals using rotary ProTaper files were done. All canals were irrigated with $10 \mathrm{~mL}$ each of $1.5 \% \mathrm{NaOCl}$ followed by saline irrigation, and a final rinse of $17 \%$ EDTA was done. The canals had no discharge, so Metapex was placed as an intracanal medicament in the same visit (Fig. 3B). The patient was discharged and asked to report after 3 months. At the third month follow-up, the patient was asymptomatic, 

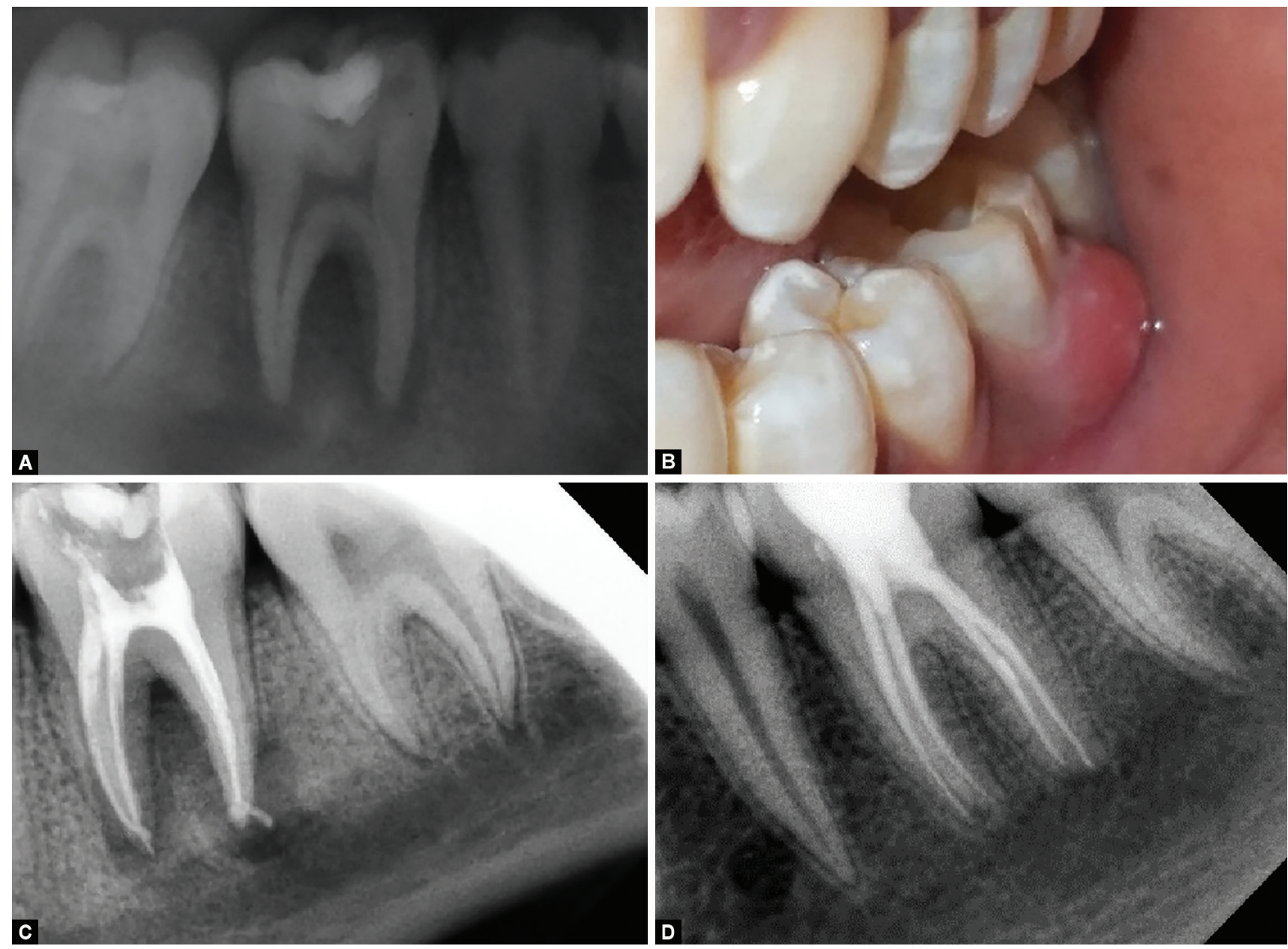

Fig. 2A to D: Case 2: (A) Preoperative Clinical photograph; (B) Preoperative Intraoral periapical radiograph; (C) Metapex placed; (D) Obturation done

and radiographic evidence of healing of the periapical lesion was confirmed. So, obturation with single-cone obturation technique was completed after complete removal of Metapex (Fig. 3D).

\section{Case 4}

A 15-year-old male patient reported to the Department of Conservative Dentistry and Endodontics with a chief complaint of a broken tooth in the lower front teeth region for the past few months. Clinical examination showed the presence of gingival polyp over the carious tooth structure, and IOPA revealed radiolucency involving enamel, dentin, and pulp associated with a periapical radiolucency in 42 that was found to spread to the surrounding apical region of 41 of size $3 \times 5 \mathrm{~mm}$ (Fig. 4A). Pulp sensibility tests produced no response in these teeth. It was diagnosed as asymptomatic irreversible pulpitis with a gingival polyp in 42 associated with apical periodontitis in 41 and 42 . Nonsurgical endodontic therapy with Metapex was planned for 41 and 42.

After LA administration, under dam isolation, gingival polyp was excised in 42, and access was opened in both 41 and 42 . Complete cleaning and shaping of the canals using the step-back technique were done. Both teeth were irrigated with $10 \mathrm{~mL}$ each of $1.5 \% \mathrm{NaOCl}$ followed by saline irrigation, and a final rinse of $17 \%$ EDTA was done, and closed dressing was given in the first visit. In the subsequent visit, Metapex was placed as an intracanal medicament (Fig. 4B). The patient was advised to report after 3 months. The patient returned after 3 months and was asymptomatic, and complete resolution of the periapical lesion in 41 and 42 was noted. Metapex was completely removed from the teeth, and obturation with cold lateral condensation technique was done (Fig. 4D).

\section{Discussion}

Necrotic pulps act as nidi for microbial growth. These organisms subsequently release toxins into the periapical region that trigger the inflammatory reaction and bone resorption. ${ }^{3}$ Inflammatory lesions of endodontic origin may vary in size from 5 to $8 \mathrm{~mm}$ in diameter. Up to 10-mm diameter size, the lesions are considered as granulomas while larger ones as cysts. Different nonsurgical and surgical procedures can be chosen for managing the lesions of endodontic origin. Cleaning and shaping of the root canal and microbial eradication are the key factors in achieving a successful outcome nonsurgical root canal treatment should be done at first, which has 42 to $74 \%$ of healing of lesions. ${ }^{2}$

In younger individuals, surgical choices are considered unpleasant and more aggressive than nonsurgical endodontic 

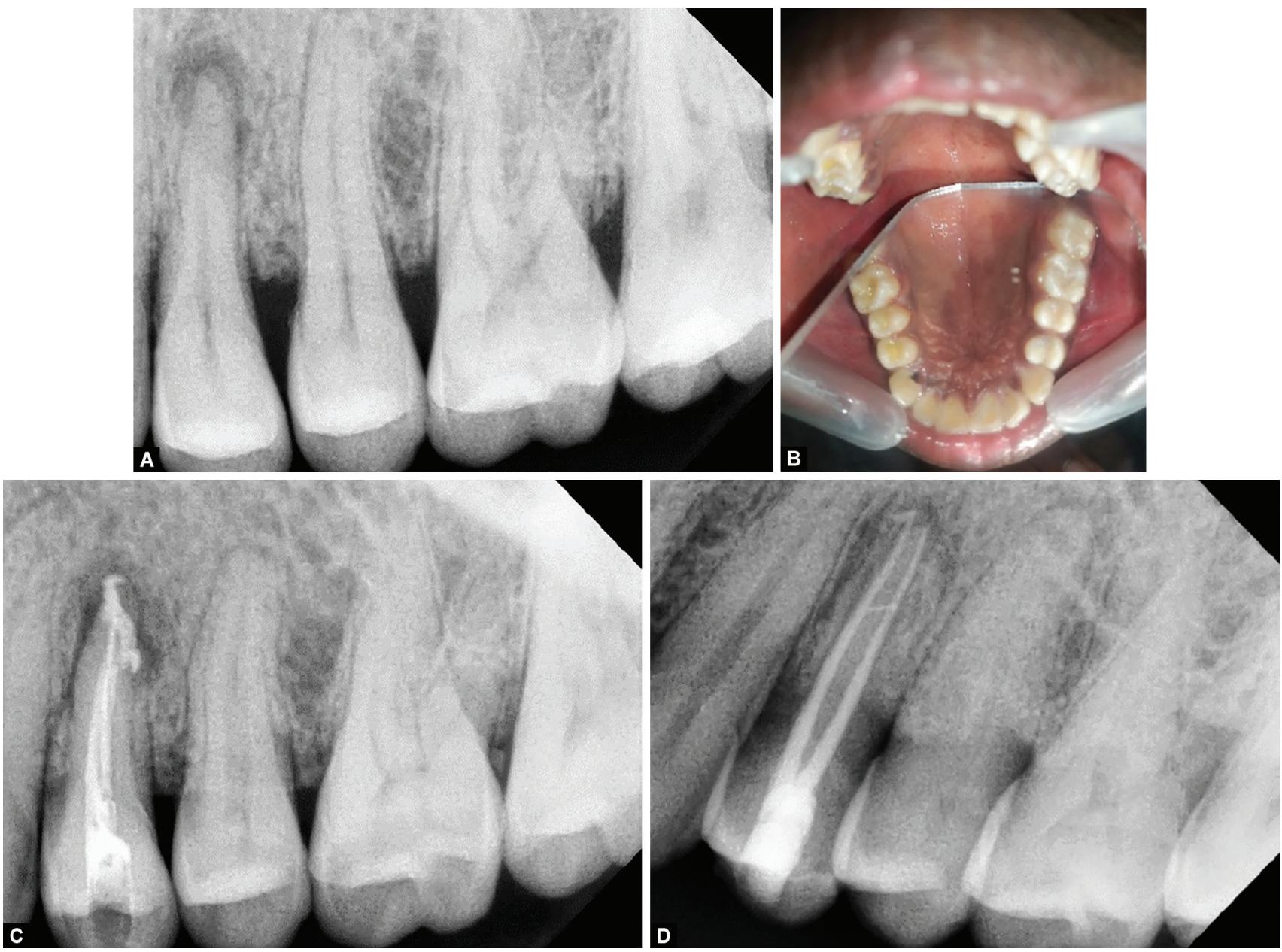

Fig. 3A to D: Case 3: (A) Preoperative clinical photograph; (B) Preoperative intraoral periapical radiograph; (C) Metapex placed; (D) Obturation done

treatment. Further, root resection would compromise the crownto-root ratio. These drawbacks can be overcome in a nonsurgical approach that promotes the healing and maturation of young permanent teeth. ${ }^{7}$ Further, the less-invasive nature of such procedure reduces psychological trauma and increases patient compliance. Moreover, in the better-perfused tissue with a greater number of undifferentiated cells and excellent lymphatic drainage, the lesion can be eliminated by capitalizing on the removal of the etiological factors by endodontic treatment and greater healing ability of periradicular tissues. ${ }^{2}$

Due to its better disinfection potential, $\mathrm{Ca}(\mathrm{OH})_{2}$-based paste was used as an intracanal medicament in all these cases. As the periapical tissues can naturally heal, treatment of lesions of endodontic origin should focus on only eliminating the etiological agents. ${ }^{3}$ In all these cases, resolution of the lesion 3 months after medicament placement was confirmed. Radiographical assessment of bone healing was evidenced by increased density, trabecular reconstruction, and lamina dura formation. Coronal leakage could compromise prognosis and hence should be avoided. So after obturation, bonded composite restorations were placed immediately.

$\mathrm{CH}$ paste was selected as the intracanal medicament as there is ample literature evidence for its ability to heal periapical inflammation and form apical calcific barrier. ${ }^{7}$ To effectively eliminate bacteria in the dentinal tubules, intracanal medication with $\mathrm{CH}$ should remain in the canal for at least seven days. The antibacterial effect is due partly to its high $\mathrm{pH} 12$, which prevents the growth and survival of many oral bacteria. $\mathrm{CH}$ disrupts the microbial cell wall lipopolysaccharides in gram-negative organisms and hampers membrane transport mechanisms, resulting in cell death. The primary advantage of $\mathrm{CH}$ is its ability to kill microorganisms in the absence of direct contact by absorbing the $\mathrm{CO}_{2}$ required for bacterial growth and by the release of hydroxyl ions, which diffuse into dentinal tubules. ${ }^{6} \mathrm{CH}$ paste affects periapical healing and repair by eradicating residual microorganisms, controlling inflammation, stimulating calcific tissues, counteracting acidic $\mathrm{pH}$ of osteoclasts, and neutralizing endotoxins. ${ }^{2}$

Even though $\mathrm{CH}$ is largely effective as an intracanal medicament, certain microbial species, like Enterococcus faecalis, are reported to be resistant to its effects. E. faecalis is difficult to remove during retreatment, and the retreatment fails more if $E$. faecalis is cultivable at the time of obturation. To improvize the $\mathrm{CH}$, synergistic and additive antimicrobial agents with specific chemical characteristics have served as suitable vehicles. ${ }^{6}$

A study comparing the effect of $\mathrm{CH}$ and its combination with various vehicles showed that at the dentinal depth, $\mathrm{CH}$ alone 

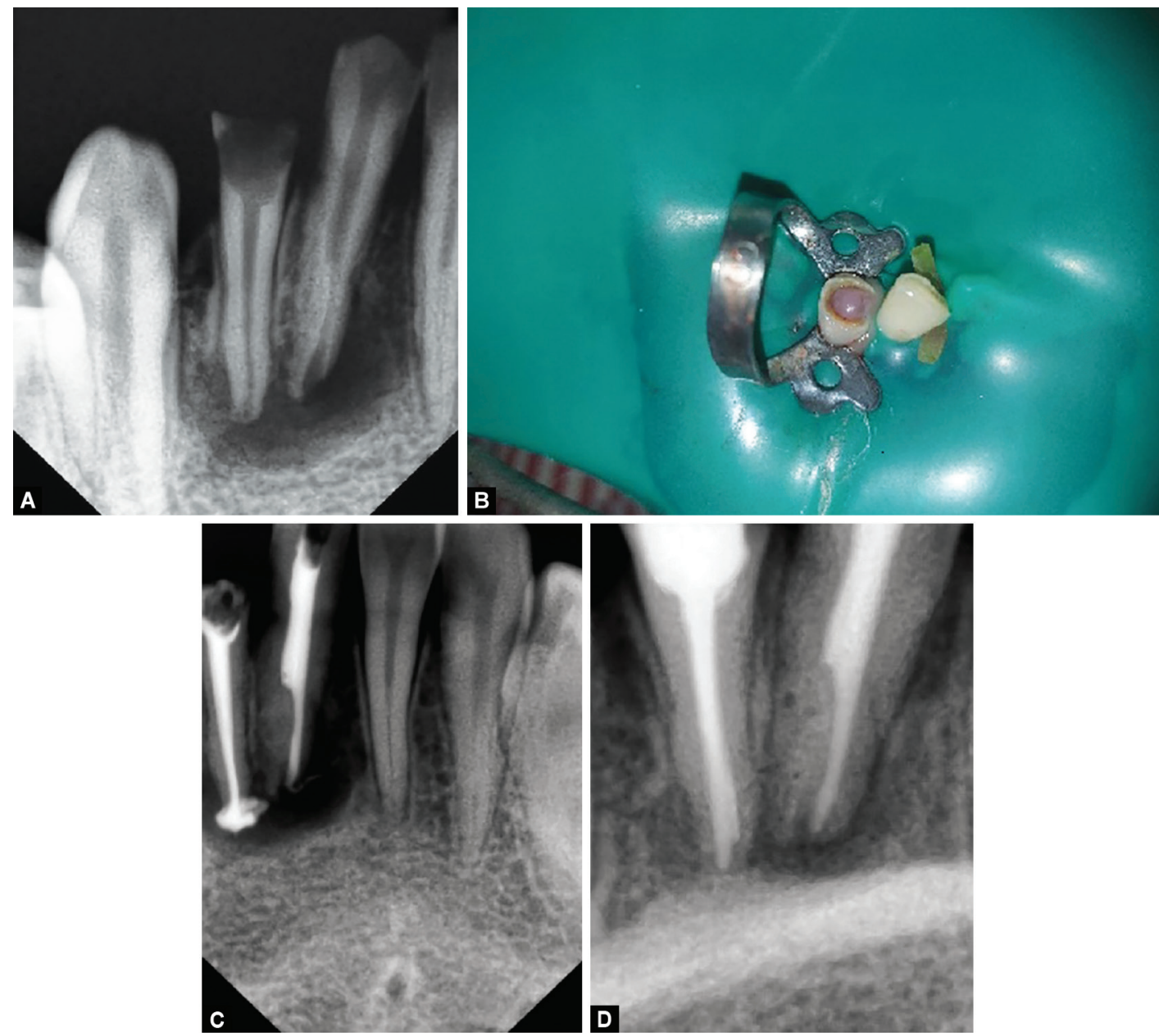

Fig. 4A to D: Case 4: (A) Preoperative clinical photograph; (B) Preoperative intraoral periapical radiograph; (C) Metapex placed; (D) Obturation done

may not diffuse deep into the tubules and hence resulted in significantly higher numbers of E. faecalis at a depth of $250 \mu \mathrm{m}$. This may be due to the inability of the $\mathrm{CH}$ paste to effectively penetrate deep into the dentinal tubules, resulting in sublethal doses of antimicrobial agents at these greater depths. E. faecalis is killed at $\mathrm{pH}$ levels higher than 11.5. $\mathrm{CH}$ has a $\mathrm{pH}$ of $\sim 12$; due to the dentin's buffering action, $\mathrm{pH}$ may not be maintained high at deeper tubules, helping E. faecalis to thrive. E. faecalis effectively penetrates up to $300 \mu \mathrm{m}$ into the dentinal tubules, with significantly less bacteria being found at greater dentinal tubule depths.

Metapex, which contains $60 \% \mathrm{CH}$ and $38 \%$ iodoform in silicone oil, is widely used. The antimicrobial action of it was evident at deeper tubules. Its superior antibacterial effects were demonstrated at dentinal tubule depths even up to $250 \mu \mathrm{m}$. Metapex contains silicone oil as its vehicle and has a $\mathrm{pH}$ below that which is effective to kill $E$. faecalis. The greater antimicrobial action of Metapex could be due to the presence of iodoform and the oily vehicle that increases the availability of the active agent for a prolonged duration. This increases the eradicative effect on E. faecalis and other bacteria. Not only is iodoform effective as a medicament, it has also been shown to be host-tissue friendly and hence often used as a resorbable medicament in primary pulpectomies also. ${ }^{6}$

\section{CONCLUSION}

In all these cases, extensive periradicular lesions were treated with Metapex as intracanal medicament and endodontic treatment. This confirms that inflammatory lesions of endodontic origin can heal by nonsurgical interventions.

\section{Clinical Significance}

For the treatment of periapical lesions, nonsurgical therapy should be considered as first-line treatment option as it proves to be a more conservative approach.

\section{References}

1. Dubey $S$, Dhole $T$, Boruah $L$, et al. Comparative antimicrobial efficacy of Metapex, Metronidazole, BioPure MTAD, Aztreonam on Bacteroides fragilis and Propionibacterium acne. J Conserv Dent 2013;16(4):327. DOI: 10.4103/0972-0707.114361.

2. Ghorbanzadeh S, Ashraf H, Hosseinpour S, et al. Nonsurgical management of a large periapical lesion: a case report. Iran Endod J 2017;12(2):253-256. DOI: 10.22037/iej.2017.49.

3. Sood N, Maheshwari N, Gothi R, et al. Treatment of large periapical cyst like lesion: a noninvasive approach: a report of two cases. Int J Clin Pediatr Dent 2015;8(2):133-137. DOI: 10.5005/ jp-journals-10005-1299. 
4. Fernandes M, De Ataide I. Non-surgical management of a large periapical lesion using a simple aspiration technique: a case report. Int Endod J 2010;43(6):536-542. DOI: 10.1111/j.1365-2591.2010. 01719.x.

5. Fernandes $\mathrm{M}$, Ataide I. Nonsurgical management of periapical lesions. J Conserv Dent 2010;13(4):240. DOI: 10.4103/0972-0707.73384.
6. Cwikla $\mathrm{S}$, Belanger $\mathrm{M}$, Giguere $\mathrm{S}$, et al. Dentinal tubule disinfection using three calcium hydroxide formulations. J Endod 2005;31(1):5052. DOI: 10.1097/01.don.0000134291.03828.d1.

7. Ogonji G. Non-surgical management of a chronic periapical lesion associated with traumatised maxillary central incisors: case report. East Afr Med J 2004;81(2):108-110. DOI: 10.4314/eamj.v81i2.9135. 